\title{
Effective Practice Using Pole Exercise Would Contribute for The Situation of "Exercise is Medicine"
}

\author{
Hiroshi Bando, MD, PhD, FACP $^{1,2^{*}}$, Akito Moriyasu ${ }^{3,4}$, Mitsuru Murakami ${ }^{5}$ \\ ${ }^{1}$ Tokushima University / Medical Research, Tokushima, Japan \\ ${ }^{2}$ Japan Masters Athletics, Tokushima division, board, Tokushima, Japan \\ ${ }^{3}$ Rehabilitation Research Group for body and heart in Shikoku, Kagawa, Japan \\ ${ }^{4}$ Akiboshi Bright Star training rehabilitation center, Kagawa, Japan \\ ${ }^{5}$ Japan Masters Athletics, Kagawa division, vice-president, Kagawa, Japan
}

DOI: $10.36348 /$ jaspe.2020.v03i08.004 | Received: 30.07.2020 | Accepted: 06.08.2020 | Published: 09.08.2020

*Corresponding author: Hiroshi BANDO, MD, PhD, FACP

\section{Abstract}

The presence of the sports can historically contribute international understanding and cooperation. Olympic and Paralympic Games in Tokyo will be scheduled, and "Exercise is Medicine" has been known widely by American College of Sports Medicine (ACSM). Authors et al. have continued clinical practice and research on pole exercise for years. The methods include various movements in frontal, horizontal and sagittal planes. The pole consists of aluminum with 60-160 $\mathrm{cm}$ in length. Moriyasu pole training would be clinically effective, which can simultaneously increase the flexibility of thorax, vertebrae and pelvis, and the functioning ability of joint movement and posture control.

Keywords: Exercise is Medicine, pole exercise, physical flexibility, American College of Sports Medicine (ACSM).

Copyright @ 2020: This is an open-access article distributed under the terms of the Creative Commons Attribution license which permits unrestricted use, distribution, and reproduction in any medium for non-commercial use (NonCommercial, or CC-BY-NC) provided the original author and source are credited.

\section{INTRODUCTION}

Historically speaking, the presence of the sports can be a major force for international understanding and cooperation. Olympic and Paralympic Games in Tokyo will be scheduled for summer of 2021 [1]. If a person has always committed some sports related to daily life, one seems to have a precious long life in the future. From sports and rehabilitation points of view, "Exercise is Medicine" has been known widely [2]. American College of Sports Medicine (ACSM) has emphasized the importance of exercise so far. It can be prescribed just like oral medicine, and can be recommended for adequate time, place and occasion for all people [3].

Authors and colleagues have reported various research concerning sports medicine, rehabilitation, workshops for the elderly, professional athletes, masters' athletes, high school athletes, and others [4-6]. Among them, several researches were shown on the movements of the connection of the head, chest and pelvis [7]. These parts may have mutual influence like connected three spheres. Furthermore, we have continued several investigations of function in the thorax, vertebrae and lumbar region, which means the trunk [8]. As to the clinical function of the trunk, we have investigated for years by the useful method of pole exercise.

There has been a variety of discussion concerning the stability of the spine [9]. Human body has changing postures and movements, and has mechanisms for active, passive and neural control subsystem. It is necessary to prevent spinal disorders and control spinal function [10].

Pole exercise was originally begun by the author Moriyasu several years ago. Moriyasu pole is light in weight and made of aluminum with four parts. It can be connected freely, and its length can be set from $60 \mathrm{~cm}$ to $160 \mathrm{~cm}$. Figure 1 shows the general information of how to use the pole. We can make stretch our body in three dimensions including frontal plane, horizontal plane and sagittal plane, respectively (Fig.1a, b, c). We can move our body not only in each plane, but also in combined 2-3 dimensions by the spiral and rotation movements. We have our basal protocol of the movements, which has six recommended methods for everyone [5]. They are i) lateral bending, ii) chest axis rotation, iii) lumbar lordosis and pelvic anteversion, iv) movement for kyphosis of thoracic vertebra, v) spine pelvic axis rotation, vi) warp and rounding of the back. 
In one of our previous reports, the intervention of pole exercise was performed for physical flexibility and exercise function [5]. Before and after the intervention, evaluation was conducted by 11 tests. Tests of physical flexibility included Thomas Test, Wing Test, Shoulder Extension Test, straight leg raise (SLR), Body warp prone position, Finger Floor Distance (FFD) and Heel Buttock Distance (HBD). Tests of exercise functions included Functional Reach Test (FRT), Back Muscle Strength (BMS), Weight Bearing Index (WBI) and closed eye leg standing time. As a result, there were significant improved functions between before and after the intervention $(p<0.05)$. Consequently, adequate pole exercise may give beneficial human abilities in physiotherapy, especially physical flexibility and exercise function in short period [11].

This training using pole exercise has been applied to subjects and patients with various diseases or targets. They include elderly people, patients with orthopedic illness, and athletes from various sports. Through pole practice continuation, we have found clinical efficacy of physical and mental functions. There are three possible reasons in the following.

The first reason would be the presence of motion segments [12]. Trunk has thorax (rib cage), vertebrae (spinal column) and pelvis. Among them, the function of the spinal column is like a pillar, which is composed of 24 vertebrae. In fact, the trunk moves subtly during some exercise. At that time, 24 vertebrae can work its function collectively as one unit. On the other hand, each segment is responsible for its own function and sometimes moves in coordination [13]. In the case of the motor dysfunction of the spinal column, it is rare that only one segment is damaged. Usually, the cause of the impaired function would be from several motion segments. The segments sometimes move excessive or suppressed manners.

The second reason would be the improved mobility in the thorax [14]. Since thorax occupies about $40 \%$ of the weight mass of the trunk, it is considered that the thorax may be regarded as one mass. However, it is not a single rigid block, but a series of materials where every point can move subtly. In other words, it can always move freely because there are many synovial joints such as facet joints and costal joints. As a matter of fact, thorax has the function of articulation by two mechanisms. They are the axial rotation movement of the costo-capital joint and the glenocaudal sliding movement of the transverse rib joint. Consequently, both movements are always observed simultaneously [14].

The third reason would be the availability of pole exercise where the subject can perform in any postures [15]. The exercise has been conducted in upright position, half-sitting position with reclining to a bar or sitting position on the chair. If the patient cannot stand, he can sit on the floor and receive specific pole exercise for rehabilitation procedure. Furthermore, Moriyasu pole can be set from short to long length according to the different kinds of pole exercise.

There is some limitation of our clinical practice and research [16]. As regards to many patients with orthopedic disorders or sports injuries, they usually have not only one problem, but several complicated health and medical problems [17]. Consequently, our staffs have to understand the background and evaluate the general situation.

In summary, we have continued various practice and research on pole exercise. Pole Training can simultaneously increase the flexibility of thorax, vertebrae and pelvis, and the functioning ability of joint movement and posture control. This article will become hopefully one of the references in the region of sports medicine and rehabilitation.

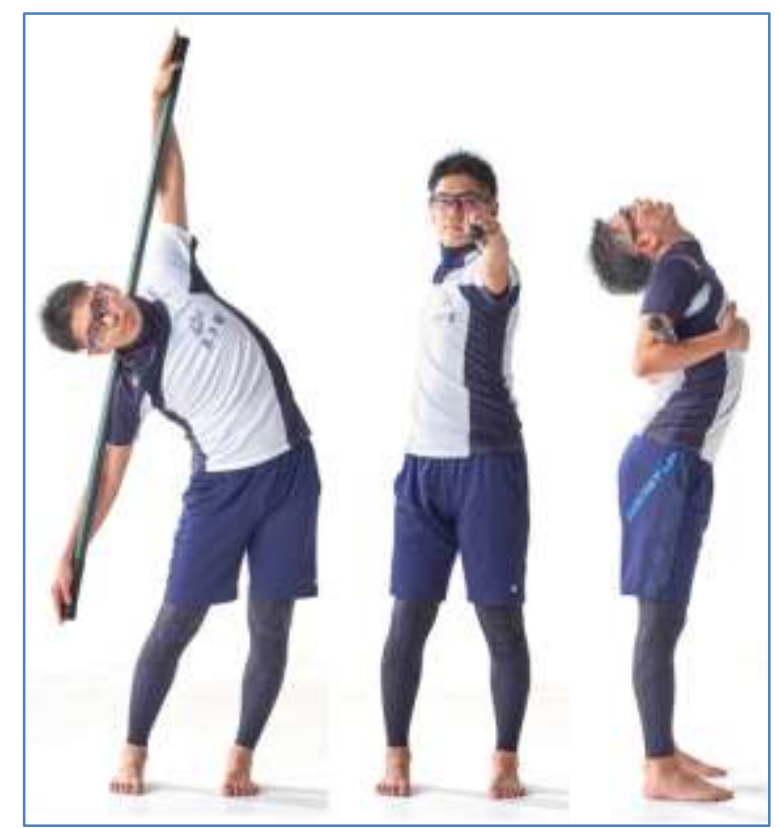

Fig-1: Three-dimensional movement for pole exercise

Fig.1a movement in frontal plane

Fig.1b movement in horizontal plane

Fig.1c movement in sagittal plane

\section{ACKNOWLEDGMENT}

Authors express our gratitude for related people concerning this study.

\section{CONFLICTS OF INTEREST}

The authors declare no conflict of interest.

\section{REFERENCES}

1. Tokyo Olympic and Paralympic.
https://tokyo2020.org/en/
2. Lobelo, F., Stoutenberg, M., \& Hutber, A. (2014). 
The exercise is medicine global health initiative: a 2014 update. British journal of sports medicine, 48(22), 1627-1633.

3. American College of Sports Medicine (ACSM). Riebe D, Ehrman JK, Liguori G (eds). (2017). ACSM's guidelines for exercise testing and prescription $10^{\text {th }}$ ed. Lww, Philadelphia.

4. Murakami, M., \& Bando, H. (2018). Forward leaning and two axis operation for effective and safe running. Sports Med Rehabil J. 2018; 3 (4), 1042.

5. Moriyasu, A., Bando, H., Murakami, M., Inoue, T., Taichi, A., Wakimoto, K., ... \& Akayama, R. (2018). Pole exercise causes body changes in physical flexibility and exercise function. Journal of Novel Physiotherapies, 8(1), 377.

6. Bando, H., Moriyasu, A., \& Murakami, M. (2020). Recent Trends of Common Factors of Geriatric Medicine and Rehabilitation Medicine. J Health Med Res, 2(1), 1-3.

7. Murakami, M., Bando, H., \& Moriyasu, A. (2020). The Concept of Trunk Connection can be Applied for the Training of Short Distance Sprint. Journal of Sports Medicine and Rehabilitation, 1(1), 001.

8. Bando, H., \& Murakami, M. (2018). The important points in plantar region for relaxed standing and running. Res Rev Orhop, 2(2), 7-9.

9. Panjabi, M. M. (1992). The stabilizing system of the spine. Part I. Function, dysfunction, adaptation, and enhancement. J Spinal Disord, 5(4):383-9

10. Roberto, I., Gianluigi, G., Guglielmi, G., \& Mario,
M. (2012). Biomechanics of the spine. Part II: Spinal instability.

11. Kurihara, R., Fujimoto, D., Dakeshita, T., Moriyasu, A., \& Bando, H. (2019). The influence of Pole exercise on the range of motion of thoracic spine. Clinical Research in Orthopaedics, 2(1).

12. Wilke, H. J., Grundler, S., Ottardi, C., Mathew, C. E., Schlager, B., \& Liebsch, C. (2020). In vitro analysis of thoracic spinal motion segment flexibility during stepwise reduction of all functional structures. European Spine Journal, 29(1), 179-185.

13. Galbusera, F., \& Bassani, T. (2019). The spine: a strong, stable, and flexible structure with biomimetics potential. Biomimetics, 4(3), 60.

14. Lee, N. G., You, J. S. H., Kim, T. H., \& Choi, B. S. (2015). Intensive abdominal drawing-in maneuvers after unipedal postural stability in nonathletes with core instability. Journal of Athletic Training, 50(2), 147-155.

15. Moriyasu, A., Bando, H., \& Murakami, M. (2019). Pole exercise would be clinically effective through increasing thoracic spinal mobility. Journal of Physical Medicine and Rehabilitation, 1(1), 03.

16. Cruz-Jimenez, M. (2017). Normal changes in gait and mobility problems in the elderly. Physical Medicine and Rehabilitation Clinics, 28(4), 713725.

17. Frost, B. A., Camarero-Espinosa, S., \& Foster, E. J. (2019). Materials for the spine: anatomy, problems, and solutions. Materials,12(2), 253. 